BULLETIN Bulletin hispanique

HISPANIQUE Université Michel de Montaigne Bordeaux

123-2 | 2021

Investigaciones semánticas y léxicas actuales

\title{
Marine Poirier, La coalescence en espagnol. Vers une linguistique du signifiant énactivisante. Préface de Didier Bottineau
}

Limoges : Lambert Lucas, 2021

Élodie Blestel

CpenEdition

Journals

Édition électronique

URL : https://journals.openedition.org/bulletinhispanique/14622

DOI : 10.4000/bulletinhispanique. 14622

ISBN : $1775-3821$

ISSN : $1775-3821$

Éditeur

Presses universitaires de Bordeaux

Édition imprimée

Date de publication : 20 décembre 2021

Pagination : $396-403$

ISBN : $979-10-300-0-745-9$

ISSN : 0007-4640

Référence électronique

Élodie Blestel, « Marine Poirier, La coalescence en espagnol. Vers une linguistique du signifiant énactivisante. Préface de Didier Bottineau », Bulletin hispanique [En ligne], 123-2 | 2021, mis en ligne le 20 décembre 2021, consulté le 07 février 2022. URL : http://journals.openedition.org/ bulletinhispanique/14622 ; DOI : https://doi.org/10.4000/bulletinhispanique.14622

Ce document a été généré automatiquement le 7 février 2022.

Tous droits réservés 


\section{Marine Poirier, La coalescence en espagnol. Vers une linguistique du signifiant énactivisante. Préface de Didier Bottineau}

Limoges : Lambert Lucas, 2021

Élodie Blestel

\section{RÉFÉRENCE}

Marine Poirier, La coalescence en espagnol. Vers une linguistique du signifiant énactivisante. Préface de Didier BOTTINEAU - Limoges, Lambert Lucas, 2021, 320 pages. ISBN/EAN

978-2-35935-341-9

1 Ce premier et passionnant ouvrage de Marine Poirier, La coalescence en espagnol. Vers une linguistique du signifiant énactivisante (Lambert-Lucas, 2021) se donne pour objectif de mettre au jour les mécanismes qui président à ce que l'autrice identifie comme la « coalescence » d'éléments réputés discrets en diachronie de l'espagnol (tan bien, cual quier, toda vía...) et dont la fusion graphique (también, cualquier, todavía...) s'accompagne de changements syntaxiques et sémantiques que les linguistes ne sont pas en mesure d'expliquer à l'aune des propriétés propres aux unités fusionnées envisagées isolément (i.e. dans un découpage morphématique traditionnel) ${ }^{1}$.

2 Pour dépasser et résoudre cette difficulté, Marine Poirier avance une proposition aussi inventive qu'originale : les signifiants unitaires nouvellement obtenus par coalescence résultent d'un processus de resegmentation, par l'interprétant, via une réanalyse analogique et submorphémique de la séquence vocale en sandhi émergeant du copositionnement initial ${ }^{2}$. Pour appréhender ce mécanisme, il appartient dès lors au linguiste d'examiner «le magma » de la parole (p.162), cette dernière étant conçue comme expérience, geste, action et processus vocal «à segmenter et à sémiotiser » 
(ibid.). C'est ainsi que, à partir de ce qu'elle appelle cette nouvelle "primitive observable» (p. 152) - à savoir, la parole dans ses dimensions processuelle et motrisensorielle -, la chercheuse s'emploie à reconstruire ce qui a pu, dans la structure et la syntaxe de ces signes incidemment copositionnés, être relu et reconnu de manière inédite et innovante, qui a fini par aboutir à la «lexicalisation-canonisation» (p. 112) d'un nouveau signifiant unitaire (moyennant une certaine routinisation préalable).

Cette hypothèse de resegmentation analogique et submorphémique des signifiants copositionnés en sandhi par l'allocutaire représente un éclairage précieux et particulièrement convaincant sur le processus de création néologique que constitue la coalescence, et, plus largement, sur les mécanismes à l'œuvre dans le changement linguistique. Mais les observations de la chercheuse invitent aussi à repenser toute notre conception du langage et de son fonctionnement à la lumière de ces mécanismes. C'est ce qui fait, à mon sens, tout le sel de cet ouvrage. Car Marine Poirier ne se contente pas d'apporter une élégante - et éloquente - explication au phénomène de la coalescence en espagnol, elle s'atèle ce faisant à une difficile et exigeante mise au point épistémologique pour laquelle elle fait preuve d'une écoute théorique, d'une distance critique et d'un esprit de synthèse des plus remarquables : de la conception du signe chez Saussure, Martinet ou Benveniste (entre autres), aux apports les plus récents de la psychologie et des sciences cognitives contemporaines, tout est scruté, pesé, pensé et (ré)analysé dans l'optique de l'édification d'un appareil théorique renouvelé et minutieusement élaboré. Aussi, les réflexions et propositions conceptuelles offertes au lecteur dans cet ouvrage vont-elles bien au-delà de ce pour quoi elles ont été pensées initialement et constitueront sans aucun doute une référence durable dans le champ de cette très judicieusement nommée Linguistique du Signifiant Énactivisante (LSE).

L'ouvrage, préfacé par Didier Bottineau (p.9-15)3, s'ouvre sur un «rapprochement curieux » (p. 17) - qui s'éclaire pourtant très vite à mesure que se déploie la thèse de ce livre - : celui établi entre des resegmentations de chaînes signifiantes telles que jamás (< ya más), también (< tan bien) ou todavía (< toda vía), par exemple, et les «figures ambigües » chères à la Gelstattheorie, dont l'une d'entre elles - le bois gravé d'Escher, Canards et poissons, ou L'air et l'eau (1938) - orne opportunément la couverture du volume. Rapprochement curieux, donc, et pourtant puissamment éclairant, tant il illustre un principe clé de la thèse qui va être avancée : le sens de ces figures ambigües, tout comme celui des coalescences étudiées, résulte des expériences qui en sont faites par les interprétants. C'est donc avec cette belle et prometteuse analogie que la chercheuse annonce sa démarche, qu'elle résume comme une approche "phénoménogénétique " du signifiant pour laquelle plutôt que de prendre le signifiant comme un donné, il s'agit d'interroger ses conditions de constitution, enracinées dans l'expérience qui en est faite par l'interprétant : une expérience matérielle et ancrée dans la temporalité de la phrase (p. 27).

5 Dans ce dessein, Marine Poirier inscrit sa pensée dans une double filiation : celle de la Linguistique du signifiant - dont les postulats reposent sur les principes de la biunivocité des deux faces du signe, la motivation relative et la primauté accordée au signifiant dans l'engendrement du sens ${ }^{4}$-, et le paradigme de l'énaction initié par Varela et Maturana ${ }^{5}$ en sciences cognitives; deux héritages théoriques, donc, que, dans les pas de Bottineau (2010, 2016, 2017a, 2018), la chercheuse entend articuler avantageusement. pré-donné, la cognition est l'avènement conjoint d'un monde et d'un esprit ${ }^{6}-$, la 
chercheuse se les applique d'abord et avant tout à elle-même : consciente de ce qu'en tant qu'analyste et "observatrice $»^{7}$ des faits langagiers, elle prend toute sa part dans l'avènement, voire la "distinction », au sens maturanien du terme, de la coalescence en tant que phénoménologie (p.34), elle consacre la première partie de son ouvrage à en «[c]onstruire l'objet » (p. 35-117). Ainsi, après avoir rappelé les critères selon lesquels on distingue habituellement la coalescence - un procédé réputé fortuit selon Coseriu (2003) (p. 35 et ss.)-, des procédés plus systématiques de formation des mots (composition, dérivation, parasynthèse), la chercheuse s'attache-t-elle à la définir en contraste avec l'agglutination et l'amalgame, au sein desquels ce phénomène fait figure d'intermédiaire. La coalescence est ensuite utilement confrontée aux notions de "congloméré» (Benveniste) et de lexicalisation (pp.95-112), toujours dans une démarche critique des conceptions du signe et du langage que les diverses propositions théoriques parcourues révèlent. La chercheuse peut alors clore cette première partie de l'ouvrage en ayant tracé les premiers contours de son objet d'étude : i) ces coalescences sont des segments qui ont été copositionnés l'un à l'autre, ii) leur avènement bouleverse le parcours des opérations syntaxiques à l'œuvre dans la phrase et iii) c'est à la réception que se joue ce bouleversement, puisqu'il dépend de l'analyse que s'en donne l'interprétant ${ }^{8}$. Une fois le(s) problème(s) posé(s) de la sorte, le lecteur comprend le bien-fondé de l'approche qui va être exposée à la suite.

7 La deuxième partie de cet ouvrage, intitulée «Fonder une approche » (p. 123-221), en constitue le cœur théorique et conceptuel. Marine Poirier s'appuie ainsi habilement sur les nombreux débats suscités par le problème de la coalescence et de la subduction ésotérique chez Guillaume, puis dans la Linguistique du signifiant (ch. 4), pour soulever quelques points d'achoppement qui méritent d'être retravaillés ou réagencés « dans un dialogue avec le paradigme de l'énaction » (p. 121). Les divergences sur le traitement à donner à (-)y, notamment - pour lequel il a été avancé qu'il convenait d'en conserver le signifié une fois ce morphème coalescé (par exemple dans hay) - sont symptomatiques des écueils auxquels mène la conception, à ce niveau d'analyse et pour ce type de formants, de représentations discrètes (signifiant/ signifié), dans la mesure où cette approche peine à rendre compte des spécificités des « néo-signifiants » obtenus via ce processus néologique. Autant de questions qui invitent dès lors à interroger ce que recouvre la notion de "signifiant », "en tant qu'objet formalisé, muni de propriétés segmentales, variationnelles et combinatoires» (p.147), véhiculant un contenu de représentation - plus au moins dématérialisé ou subduit - stable et pré-donné. L'autrice propose plutôt d'emprunter une autre voie : celle qui consiste à envisager le signifiant, en tout cas à ce niveau d'analyse, comme un " événement » (p. 148) sensorimoteur et processuel, avec tout ce que cela suppose de réaménagements quant à la façon dont on conçoit l'émergence du sens dans ces conditions. La chercheuse soumet alors l'hypothèse selon laquelle le phénomène de la coalescence obéit à un processus de réanalyse et de réorganisation submorphémique par la perception, de la part du pôle allocutif, d'éléments analogènes ${ }^{9}$, qui ont pour effet de relier le signifiant à un réseau analogique qui va se renouvelant sans cesse (ch. 5 « Poser les questions autrement ») Le flux vocal n'est ainsi plus envisagé comme un ensemble organisé d'unités d'emblée discrètes mais plutôt comme un champ (au sens phénoménologique du terme), à (ré)organiser et (re)sémiotiser en permanence. Aussi, pour saisir le principe même de la coalescence, tout l'enjeu consiste à se départir, le temps de l'analyse, des « construits » que sont les morphèmes en copositionnement, pour replonger dans le «magma » du 
flux vocal et tenter de comprendre sur quels réseaux ont pu se fonder ces découpages alternatifs ${ }^{11}$. Autrement dit, selon les mots de Marine Poirier,

il ne s'agit pas tant de détruire les préconstruits que constituent les mots, les morphèmes, les objets perçus dans la chaîne parlée -lesquels ont, réflexivement, une robustesse que personne ne contesterait-, mais de s'en libérer le temps de l'analyse pour parvenir à percevoir autre chose. (p. 184)

8 À ce point de la démonstration, c'est tout naturellement qu'on accueille la lumineuse présentation du paradigme de l'énaction que nous livre la chercheuse, qui expose très clairement la façon dont elle fait siens quelques-uns de ses principes clé. Ainsi en est-il de la parlance - terme par lequel elle traduit le languaging ou lenguajear de Maturana -, qui renvoie à la « dynamique éthologique collective récurrente » (p. 201), dans laquelle s'inscrivent les locuteurs et "qui contribue à en modeler l'existence et l'expérience " (ibid.). Cette notion lui permet de revoir la parole et la langue sous un autre éclairage ; la première est ainsi entendue comme ce qu'elle est empiriquement, "une action corporelle qui produit des effets perceptuels susceptibles d'agir sur les êtres vivants " (p. 199). Quant à la seconde, elle n'apparaît donc plus comme « un système de formes abstraites à 'mettre en œuvre', mais [comme] un système de pratiques [que l'individu] réplique à sa manière » (p. 202). La chercheuse termine ce deuxième volet de l'ouvrage en dessinant ce qui pourrait être les quatre "phares méthodologiques pour une LSE » (p. 204) : corporéité - concevoir le signifiant en tant qu'action -, processualité - concevoir ce flux vocal comme une émergence occupant une certaine durée ${ }^{12}-$, distributivité rapportée au langage, elle implique que la langue n'est ni une "surplombance harmonieuse » (p. 209) ou intériorisée par tout un chacun comme prête à l'emploi, ni le fruit d'un locuteur seul - et situativité - nécessité d'intégrer le point de vue de l'observateur du langage dans l'étude de celui-ci.

Enfin, la troisième et dernière partie de cet ouvrage, "Interroger les mécanismes » (p. 223-277), met en œuvre les principes théoriques et méthodologiques exposés précédemment et commence par l'examen de quelques coalescences ratifiées par la diachronie (ch. 7). Ainsi, pour expliquer les nouveaux emplois de tAMBién et taMPoco, elle les rapproche de termes comme AMBos, AмBidextro, AMBivalencia, AмBiguo (en vertu de analogène AMB), avant de proposer que l'ensemble de ces signifiants a subi une " capture rhizomatique » (p. 241) via la grappe consonantique et submorphémique MP/ $\mathrm{MB}$ - ce qui en agrandit d'autant le réseau paronymique (empatar, compañero, cambiar, completo, etc. cf. p. 242), en vertu d'une séquence cognémique ${ }^{13}$ qui semble contribuer à l'amorçage de la notion de "réunion d'une dualité ou d'une opposition dans un ensemble »(p. 241 $)^{14}$. La disponibilité de cet analogène en espagnol explique, nous dit la chercheuse, que les allocutaires aient pu interpréter también et tampoco, d'abord comme les termes d'une structure corrélative (tan bien... como) puis comme un parcours syntaxique décorrélé en diachronie, nouveaux emplois modaux inclus ${ }^{15}$. L'autrice poursuit son exploration avec l'examen des nouveaux parcours syntaxiques auxquels ont pu donné lieu la reconnaissance des analogènes $\mathrm{ALK}^{16}$ et IA $^{17}$ dans les coalescences de cualquier et todavía, respectivement. L'ouvrage s'achève par un examen très concluant de cas de coalescences ludiques, créatives ou non ratifiées en espagnol (par exemple, la série sin encambio $\sim \sin$ empero $\sim \sin$ embargo) mais aussi, au passage, d'expressions en français (être au aguets > être au taquet, par exemple), ce qui ne laisse aucun doute quant à la fécondité du système explicatif qui a été soigneusement bâti et exposé tout au long de l'ouvrage. 
Qu'est-ce qu'un mot ? Un morphème ? Un signifiant ? Qu'est-ce qu'un «système », une "structure» linguistique? Quel rôle joue l'interlocution dans l'émergence du sens? D'où vient le changement linguistique ? Voilà quelques (grandes) questions, parmi bien d'autres, auxquelles cet ouvrage tente de répondre avec courage et rigueur. Ne vous fiez pas au titre: Marine Poirier ne nous guide pas vers, elle nous plonge au cœeur d'un édifice théorique, la Linguistique du Signifiant Énactivisante (LSE), qu'elle contribue largement à bâtir et dont elle illustre la portée, l'élégance et la puissance explicative ; voilà donc un ouvrage intelligent, sérieux, original et inspirant... Un ouvrage à (re)commander !

\section{BIBLIOGRAPHIE}

Bottineau, D. (2010), Language and Enaction. In J. Stewart, O. Gapenne, \& E. Di Paolo (Éds.), Enaction : Toward a New Paradigm for Cognitive Science (p. 267-305). MIT Press.

Bottineau, D. (2016), Linguistique incarnée et 'énactivisme' : Quelles collaborations possibles avec les neurosciences? In A. Rabatel, M. Temmar, \& J.-M. Leblanc (Éds.), Sciences du langage et neurosciences. Actes du colloque 2015 de l'Association des Sciences du langage (p. 211-232). LambertLucas.

Bottineau, D. (2017a), Du languaging au sens linguistique. Intellectica, 68 (Langage et énaction: corporéité, environnements, expériences, apprentissages), 19-67.

Bottineau, D. (2017b), Langagement (languaging), langage et énaction, a tale of two schools of scholars : Un dialogue entre biologie et linguistique en construction. Signifiances (Signifying), 1(1), 11-38. https://doi.org/10.18145/SIGNIFIANCES.V1I1.158

Bottineau, D. (2018), Incarnation langagière et grammaire des langues naturelles : Vers la fin d'un clivage. In J. Dokic \& D. Perrin (Éds.), Le malentendu : Une question de linguistique et de psychanalyse (p. 251-294). Vrin.

Chevalier, J.-C., Launay, M., \& Molho, M. (1984), La raison du signifiant. Modèles linguistiques, 6-2, 27-41.

Chevalier, J.-C., Launay, M., \& Molho, M. (1986a), Le fardeau. Langages, 82, 5-11.

Chevalier, J.-C., Launay, M., \& Molho, M. (1986b). Pour une linguistique du signifiant. Cahiers du CRIAR, 6, 85-99.

Chevalier, J.-C., Launay, M., \& Molho, M. (1988), Sur la nature et la fonction de l'homonymie, de la synonymie et de la paronymie. In C. Fuchs (Éd.), L'ambiguïté et la paraphrase. Opérations linguistiques, processus cognitifs, traitements automatisés (p. 45-52). Université de Caen.

Coseriu, E. (2003), Los procedimientos semánticos en la formación de palabras. Odisea, 3, 179-189.

Deleuze, G., \& Guattari, F. (1976). Rhizome. Éditions de Minuit. 
Macchi, Y. (1988), Structures lexicales et structures mentales: Mécanismes de la néologie en espagnol contemporain [Thèse de doctorat]. Université Paris IV - Sorbonne.

Macchi, Y. (2000), L'anticipation syntaxique de l'attribut. Essai de chronosyntaxe. In A. Résano (Éd.), Linguistique hispanique. Actes du VIIlème Colloque de Linguistique Hispanique (Université de Nantes, 5/6/7 mars 1998) (p. 395-413). Publications de l'université de Nantes.

Macchi, Y. (2005), Chronomorphogénèse verbale : Esquisse d'embryologie du verbe espagnol. Cahiers de linguistique analogique, 2, 153-204.

Macchi, Y. (2006), Transitivité et intransitivité : Propriétés du mot ou effets du processus phrastique ? Chronosyntaxe (VI). In G. Luquet (Éd.), Le signifié de langue en espagnol (p. 115-134). Presses Sorbonne Nouvelle.

Macchi, Y. (2018), Sculpture du sens et sens d'une sculpture. (Chronosyntaxe VIII). Chréode. Pour une linguistique du signifiant, 2, 149-191.

Maturana, H. R. (1978), Biology of Language : The Epistemology of Reality. In G. A. Miller \& E. Lenneberg (Éds.), Psychology and Biology of Language and Thought: Essays in Honor of Eric Lenneberg (p. 27-63). Academic Press. http://www.enolagaia.com/M78BoL.html.

Maturana, H. R., \& Varela, F. J. (1980), Autopoiesis and Cognition. The Realization of the Living. Reidel Publishing Company. https://doi.org/10.1007/978-94-009-8947-4

Maturana, H. R. \& Varela, F. J. (1994a), De máquinas y seres vivos. Autopoeisis : La organizacion de lo vivo (1a ed. 1972). Lumen.

Maturana, H. R., \& Varela, F. J. (1994b), L'arbre de la connaissance (F.-C. Jullien, Trad.). AddisonWesley France.

Varela, F. J., Thompson, E., \& Rosch, E. (1993), L'inscription corporelle de l'esprit. Sciences cognitives et expérience humaine. Seuil.

\section{NOTES}

1. Même en entérinant l'hypothèse d'une perte de contenu sémantique, les signifiants produits par coalescence ne constituent jamais la simple somme de leurs composants morphémiques initiaux.

2. À titre d'exemple, l'autrice propose que la séquence cual quier [kwalkjer] devenue cualquier a fait l'objet d'une réanalyse fondée sur l'analogène alk/alg (>cualquier), que l'on retrouve dans un réseau (ou "rhizome», voir infra, n. 10) de signifiants grammaticaux, soit comme élément d'augmentation des signifiants (ALGuno $\sim$ uno, ALGuien quien), soit comme élément de mise en contraste (ALGuno NINGuno). Cet analogène, qui apparaît dans sa plus minimale expression dans le signifiant ALGo, contribue, dans tous ces contextes, à amorcer la conceptualisation d'un « processus de sélection non achevé » (p. 250 et ss), commun - mais non réductible - aux signifiés de chacun des éléments du réseau.

3. On doit à ce chercheur d'avoir posé les jalons d'une linguistique énactive qui, dans le sillage du paradigme de l'énaction - fondé par les biologistes Varela et Maturana en réaction au paradigme cognitiviste (voir notamment Maturana \& Varela, 1980, 1994a, 1994b ou Varela et al., 1993) -, conçoit le langage et la grammaire des langues comme un champ d'interactivité incarné et situé dans lequel le sens linguistique doit être pensé comme un phénomène émergent plutôt que comme un pré-donné. Voir notamment Bottineau (2010, 2017a, 2017b, 2018). Nous revenons également sur ce parti-pris théorique tel que présenté par Marine Poirier infra. 
4. Les principes fondateurs de cette approche sont notamment exposés dans Chevalier et al. $(1984,1986 a, 1986 b, 1988)$ et ont donné lieu à de multiples travaux à leur suite.

5. Cf. supra, n. 3.

6. «Nous proposons le terme d'énaction [de l'anglais to enact: susciter, faire advenir, faire émerger], dans le but de souligner la conviction croissante selon laquelle la cognition, loin d'être la représentation d'un monde pré-donné, est l'avènement conjoint d'un monde et d'un esprit à partir de l'histoire des diverses actions qu'accomplit un être dans le monde » (Varela et al., 1993, p. 35, apud p. 24).

7. «[U]n observateur est un être humain, une personne, un système vivant qui, parmi d'autres opérations descriptives, peut faire des distinctions" (Maturana, 1978, p. 1446, apud p. 22) : l'autrice explique comment, dans cette optique, ces distinctions ne sont justement pas descriptives mais constitutives du monde énacté.

8. Pour ce faire, l'autrice s'appuie sur un corpus d'extraits issus de sa fréquentation de manuscrits médiévaux dans lesquels certaines fluctuations de graphies (fusions ou fissions) témoignent de la façon dont les unités copositionnées pouvaient être différemment interprétées dans les textes anciens.

9. Le terme est emprunté à Macchi et désigne des segments « de grandeur variable, allant du phonème au lexème en passant par le morphème» (Macchi, 1988, p. 45, apud p. 181) qui permettent à un signifiant d'entrer dans des réseaux analogiques avec d'autres signifiants « dont la structure est similaire par au moins un phonème en position identique » (Macchi, 1988, p. 13, ibid.).

10. Plutôt que de concevoir une langue en tant que système surplombant où tout serait prévu d'avance, l'autrice emprunte à Deleuze \& Guattari (1976) l'image des « rhizomes » attrapants par lesquels les grappes submorphémiques reconnues se feraient capturer: «Penser le système comme un rhizome, c'est non seulement penser de multiples réseaux dans lesquels un même signifiant pourrait s'intégrer selon ses différents analogènes ressentis (éventuellement à divers moments de son histoire), c'est aussi penser la mouvance à la fois du rhizome lui-même et de ses éléments» (p. 178).

11. L'autrice invite à passer "un cran en dessous" (p.184) mais on se demande si cette métaphore des profondeurs est la plus adéquate, tant elle rappelle furieusement certains principes générativistes. L'image temporelle (un avant des construits routinisés et stabilisés que sont les morphèmes), à laquelle elle recourt largement par ailleurs, est sans doute mieux à même d'illustrer son propos.

12. Elle propose notamment d'avoir recours à l'outil fourni par la chronosyntaxe (Macchi, 2000, $2005,2006,2018$ ) pour en rendre compte même si d'autres appareils conceptuels comparables ne sont pas à écarter (voir p. 207).

13. Les cognèmes sont « ces micro-signifiants élémentaires de niveau submorphémique qui, dans le cadre de réseaux d'oppositions au sein de systèmes grammaticaux, activent des microprocessus de synthèse du sens participant à la production de l'opérateur qui les intègre [...] » (Bottineau, 2016, p. 219, apud p. 179).

14. La chercheuse montre par ailleurs que cette grappe peut parfois être reconstruite a posteriori : c'est le cas de ensamblar (> ADSIMULARE).

15. Ces deux « lectures » différentes des signifiants pouvant très bien être concomitantes chez le locuteur et son allocutaire.

16. Cf. supra, n. 2.

17. Elle propose que cette succession de cognèmes (IA) véhicule la conceptualisation d'un « accès à la conscience » (p. 259). 


\section{AUTEURS}

\section{ÉLODIE BLESTEL}

Université Sorbonne Nouvelle, EA7345 CLESTHIA 